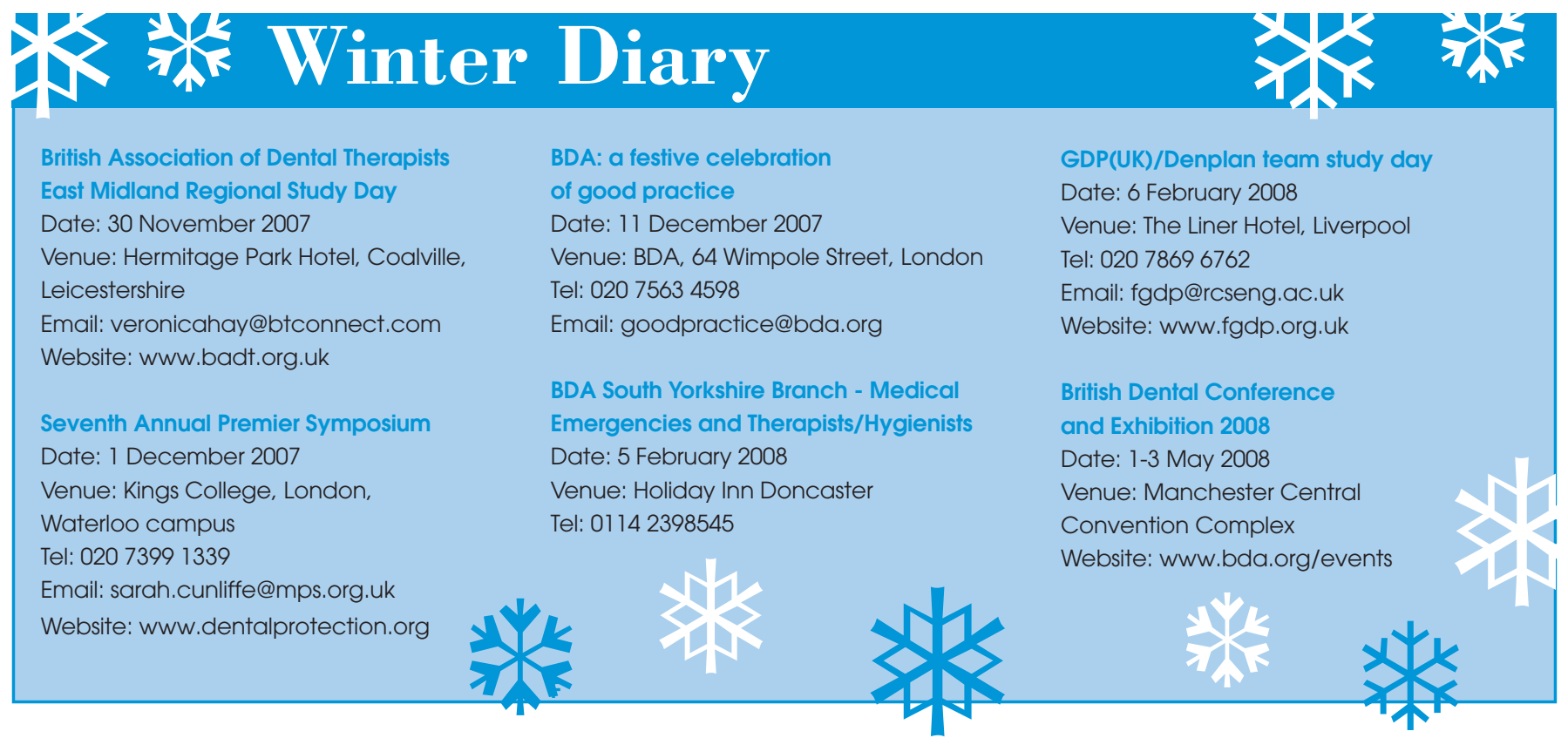

\title{
Association contacts
}

\section{BRITISH ASSOCIATION OF DENTAL NURSES}

Pam Swain (Chief Executive) PO Box 4, Room 200, Hillhouse International Business Centre, Thornton-Cleveleys, FY5 4QD Tel: $\quad 01253338360$ Email: admin@badn.org.uk Website: www.badn.org.uk

\section{BRITISH ASSOCIATION OF DENTAL THERAPISTS}

Kate Oakes (Secretary)

24 Boundary Street, Brynmawr, Gwent, NP23 4EX

Tel: $\quad 01495310946$

Email: secretary@badt.org.uk

Website: www.badt.org.uk

\section{BRITISH SOCIETY OF DENTAL HYGIENE AND THERAPY}

3 Kestrel Court, Waterwells Business Park, Waterwells Drive, Quedgley, Gloucester, GL2 2AT

Tel: $\quad 08702430752$

Website: www.bsdht.org.uk

\section{BRITISH DENTAL PRACTICE MANAGERS' ASSOCIATION}

Bridget Crump (Chairman)

3 Kestrel Court, Waterwells Drive,

Waterwells Business Park, Gloucester, GL2 2AT

Tel: $\quad 08708400381$

Email: info@bdpma.org.uk

Website: www.bdpma.org.uk

\section{DENTAL TECHNOLOGISTS ASSOCIATION}

Sue Adams (Registrar)

Waterwells Drive, Waterwells Business

Park, Gloucester, GL2 2AT

Tel: $\quad 01452886364$

Email: sueadams@dta-uk.org

Website: www.dta-uk.org

\section{ORTHODONTIC NATIONAL GROUP OF DENTAL NURSES}

Maureen Dickinson (Chairman)

Department of Orthodontics,

Scarborough General Hospital, Woodlands Drive, Scarborough, YO12 6QL

Tel: $\quad 01723342113$

Email: maureen.dickinson @acute.sney.nhs.uk

Website: www.orthodontic-ong.co.uk

\section{DENTAL LABORATORIES ASSOCIATION}

Alan Petrie (Chairman) 44-46 Wollaton Road, Beeston, Nottingham, NG9 2NR Tel: $\quad 08709914525$ Email: enquiries@dla.org.uk Website: www.dla.org.uk

\section{ORTHODONTIC TECHNICIANS' ASSOCIATION}

Paul Mallett (Secretary)

The Maxillofacial Laboratory, Royal Lancaster Infirmary, Ashton Road, Lancaster, Lancs, LA1 4RP

Website: www.orthota.co.uk

\section{CLINICAL DENTAL TECHNICIANS ASSOCIATION}

Mr Andrew McLean (Chair)

12, Upper Street North, New Ash Green, Kent, DA3 8JR

Tel: $\quad 01474879430$

Website: www.cdta.org.uk 